\title{
Ill, Itinerant, and Insured: The Top 20 Users of Emergency Departments in Baltimore City
}

\author{
Barbara Y. DiPietro, ${ }^{1}$ Dana Kindermann, ${ }^{2}$ and Stephen M. Schenkel ${ }^{3}$ \\ ${ }^{1}$ Department of Public Policy, Health Care for the Homeless, 421 Fallsway, Baltimore, MD 21202, USA \\ ${ }^{2}$ Department of Emergency Medicine, Georgetown University Hospital/Washington Hospital Center, 110 Irving Street, \\ NW, Washington, DC 20010, USA \\ ${ }^{3}$ Department of Emergency Medicine, University of Maryland School of Medicine, 110 South Paca Street, Suite 200, \\ Baltimore, MD 21201, USA
}

Correspondence should be addressed to Barbara Y. DiPietro, bdipietro@hchmd.org

Received 26 October 2011; Accepted 15 December 2011

Academic Editor: Stefano Butto

Copyright (C) 2012 Barbara Y. DiPietro et al. This is an open access article distributed under the Creative Commons Attribution License, which permits unrestricted use, distribution, and reproduction in any medium, provided the original work is properly cited.

\begin{abstract}
The purpose of this study was to document the clinical and demographic characteristics of the 20 most frequent users of emergency departments (EDs) in one urban area. We reviewed administrative records from three EDs and two agencies providing services to homeless people in Baltimore City. The top 20 users accounted for 2,079 visits at the three EDs. Their mean age was 48, and median age was 51. Nineteen patients visited at least $2 \mathrm{EDs}, 18$ were homeless, and 13 had some form of public insurance. The vast majority of visits $(86 \%)$ were triaged as moderate or high acuity. The five most frequent diagnoses were limb pain $(n=9)$, lack of housing $(n=6)$, alteration of consciousness $(n=6)$, infection with human immunodeficiency virus (HIV) $(n=5)$, and nausea/vomiting $(n=5)$. Hypertension, HIV infection, diabetes, substance abuse, and alcohol abuse were the most common chronic illnesses. The most frequent ED users were relatively young, accounted for a high number of visits, used multiple EDs, and often received high triage scores. Homelessness was the most common characteristic of this patient group, suggesting a relationship between this social factor and frequent ED use.
\end{abstract}

\section{Introduction}

Frequent emergency department (ED) users present unique challenges to clinicians and hospital administrators. Several studies have examined the impact of this patient group on ED utilization [1-4]. This population places significant economic, time, and space burdens on EDs. Patients who come to an ED more than three times per year represent about 7\% of total ED users [1]. They tend to be in poor physical and mental health, and they often come to an ED for treatment of acute medical problems [2-4]. Although the definition of "frequent user" varies greatly, distinct utilization characteristics have been noted, including a high rate of use of other parts of the health care system and visiting multiple EDs [5].

Most studies have examined the characteristics of ED visits from a macrolevel, but this study narrows the focus to the patient-specific level. We examined the details of visits made by the 20 most frequent users of three EDs in Baltimore
City in 2005 to describe the clinical and demographic characteristics of this group. The study is distinct in its attempt to look at the cooccurrence of homelessness and very high ED utilization patterns.

Approximately $1 \%$ of the US population experiences homelessness each year, and homeless patients represent about $0.4 \%$ of all ED users [6, 7]. One qualitative study from 1998 found that frequent ED users had a high prevalence of homelessness. In that study, approximately $70 \%$ of heavy users who were interviewed were homeless and many users admitted to seeking both medical and nonmedical relief during their ED visits [8].

This study seeks to more fully describe the visit details of a complex patient group, focusing on a social variable that likely contributes to high ED utilization. In particular, it strives to provide a perspective of the experience of homelessness among Baltimore City's most frequent ED users. 


\section{Methods}

This was a retrospective study based on a review of administrative records from three EDs located within 2 miles of each other and of the databases maintained by two agencies providing services to homeless people in the same urban area of Baltimore City. The most frequent users of the EDs were identified. Patients were considered homeless if they had come to at least one of the service agencies during the study year (2005). The study focuses on the 20 most frequent users identified in the combined dataset.

Each of the 20 patients was assigned a unique identifier by combining parts of the individual's social security number, date of birth, and gender and race codes. In previous research, this methodology for creating a unique identifier has been demonstrated to be compliant with requirements of institutional review boards (IRBs) and $99.8 \%$ unique to an individual [9]. These identifiers allowed patient-specific analysis of ED utilization while protecting patient confidentiality. The same methodology was used to assign identifiers to all clients of the city's location of Health Care for the Homeless during 2005 and to individuals represented in the city's centralized data system that captures utilization of publicly funded shelter beds and daytime drop-in service centers. A patient was considered homeless for the purposes of this study if his/her identifier was found in one of the homeless services administrative databases.

The top 20 most frequent users were identified based on total $\mathrm{ED}$ visits at all three institutions. Information regarding total number of ED visits, number of EDs visited, and health insurance was extracted from the ED records. Triage scores as well as the most frequently occurring ICD-9 codes listed for each patient visit were recorded. The number of visits receiving "high," "moderate," or "low" triage scores were summed, with a "high" score correlating to triage level 1 or 2, "moderate" to level 3, and "low" to level 4 or 5. Finally, the diagnostic codes for each patient were compiled and tallied across all visits for each patient.

The study was approved by the institutional review board at each participating hospital and at the university with which the lead author was affiliated at the time of the study.

\section{Results}

During calendar year 2005, the number of patient visits at the three EDs totaled 159,340. The top 20 users made 2,079 visits during this time, accounting for $1.3 \%$ of the total. Table 1 details the demographic, clinical, and housing status characteristics of these 20 patients. The average age in this group was 48.3 years, and the median age was 51 (range, 23-68). The top seven users each visited an ED at least 100 times during the year. Most of the 20 users visited all three EDs $(n=13), 6$ individuals used two EDs, and 1 went to one facility. The majority of patients had either Medicaid $(n=8)$ or Medicare $(n=4), 7$ were uninsured, and one was noted as both having Medicaid and being uninsured. The most common characteristic among these top 20 users was homelessness: 18 (90\%) had contact with homeless service agencies during the year.
For each patient, the top five most prevalent ICD-9 codes (calculated to the prime decimal point for each three-digit category) were tallied (Table 1). The ICD-9 codes indicate that some patients presented frequently for the same reasons, and others had a wide range of codes, indicating a lesser degree of consistency across visits. For example, one patient's five top diagnostic codes represented $39 \%$ of that patient's codes; for another, they represented $93 \%$ of all codes. Most patients had a relatively narrow range of diagnoses, with the top five accounting for the majority of reasons for their visits. Overall, the group's conditions span a range of acute and chronic disease (Table 2). Of the diagnoses that applied to at least 4 of the 20 patients, the most common was limb pain ( 9 patients).

\section{Discussion}

We examined the clinical and demographic characteristics of a subset of ED users who account for a disproportionately large number of total ED visits. The most frequent users are relatively young, in poor health, and experience homelessness in high numbers. The 20 patients identified in this study accounted for more than 2,000 ED visits in 2005, constituting just over $1 \%$ of the total visits for these three EDs during the study period. While our local area has housed a small number of long-term homeless individuals in a supported housing program since 2005, the general economic downturn since that time and related increase in the area's homeless population would likely make our findings more striking today.

The sheer number of visits is likely to have imposed significant time, space, and resource burdens on the EDs. A quarter of visits by the study population received high triage scores, and another $60 \%$ received moderate scores. Although triage scores are an imperfect proxy for severity of illness, this scoring pattern suggests that frequent users are relatively ill.

Most users cycled among the three EDs: 95\% visited at least two of them, and $65 \%$ used all three. The sharing of electronic medical records among the hospitals might allow better coordination of the care being delivered, reduce the possibility of conflicts in medications or discharge instructions, and limit unnecessary and repetitive workups.

The majority of the most frequent users were insured through Medicaid or Medicare; about one-third were uninsured. This belies the stereotype that frequent users are uninsured and may indicate a high incidence of disabling conditions, especially among the nonelderly population. It also implies that the Medicaid expansion in the Patient Protection and Affordable Care Act (PPACA), which targets low-income individuals, may not by itself decrease frequent ED use.

The conditions most prominent among this group of frequent ED users are acute symptoms such as pain, nausea/ vomiting, altered consciousness, and respiratory complaints. Their visits are also related to chronic diseases such as HIV infection, hypertension, drug and alcohol abuse/withdrawal, and diabetes. This combination of acute and chronic complaints indicates the importance of arranging appropriate followup to cover complex medical and behavioral health concerns as well as social challenges. 


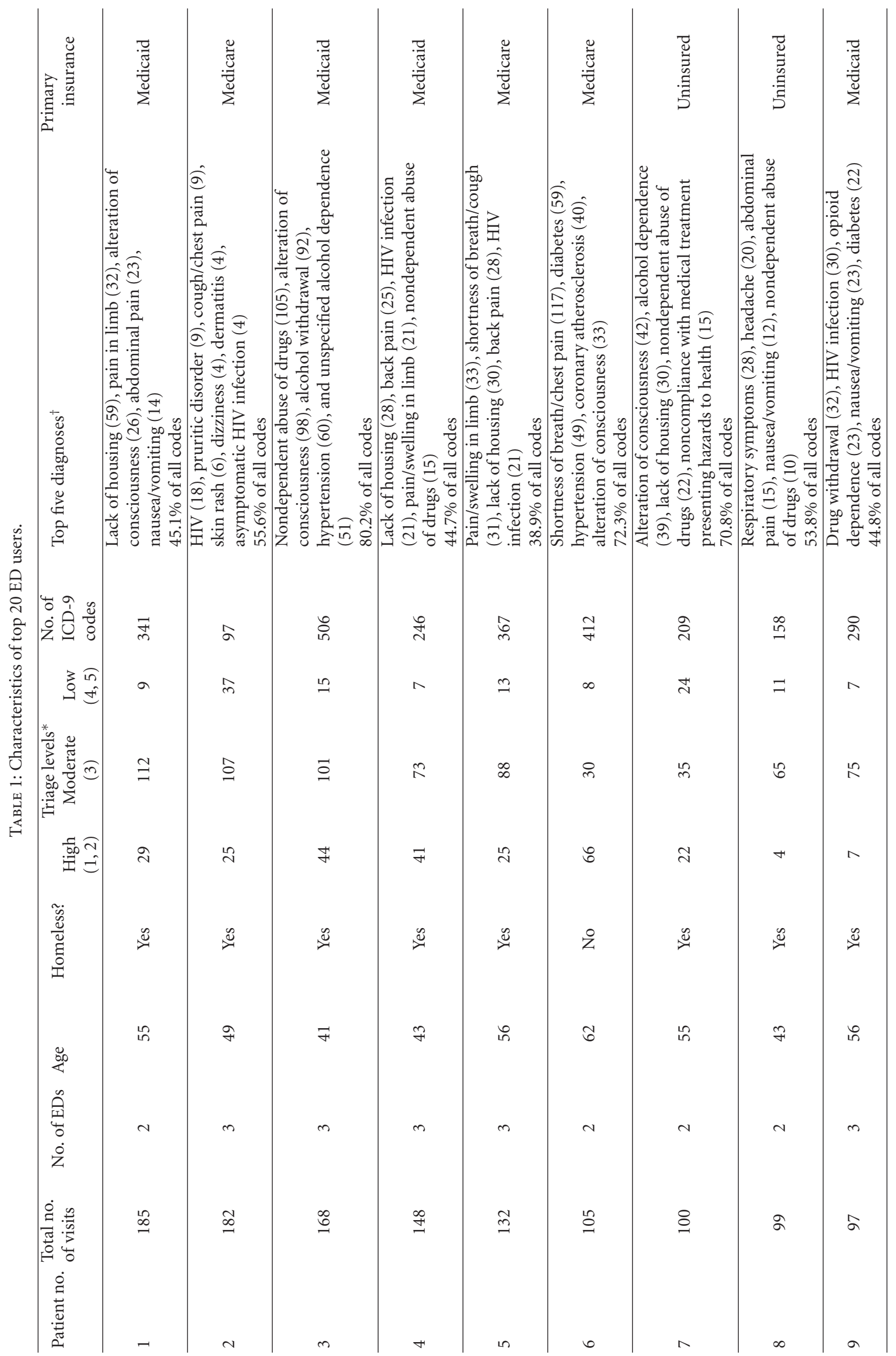




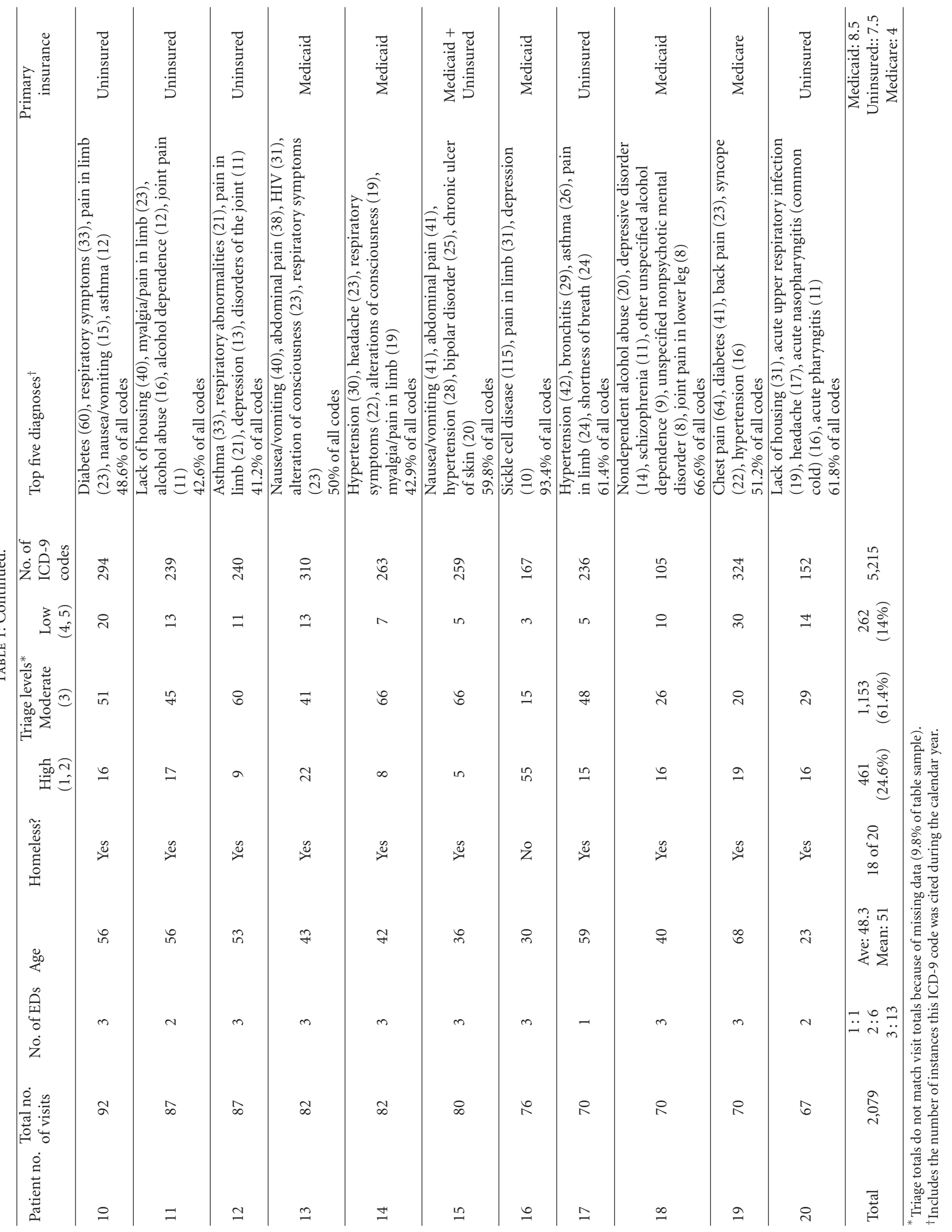


TABLE 2: Most prevalent diagnoses among 20 frequent ED users.

\begin{tabular}{lc}
\hline Diagnosis indicated by ICD-9 Code & No. of patients \\
\hline Limb pain & 9 \\
Lack of housing & 6 \\
Alteration of consciousness & 6 \\
HIV infection & 5 \\
Nausea/vomiting & 5 \\
Hypertension & 5 \\
Drug abuse & 5 \\
Alcohol abuse/withdrawal & 4 \\
Diabetes & 4 \\
Abdominal pain & 4 \\
Respiratory symptoms & 4 \\
\hline
\end{tabular}

Homelessness was the most consistently unifying characteristic among these 20 patients. Interestingly, when ICD-9 codes were used to screen for homelessness, lack of housing was found to be one of the top five diagnoses in six patients. When these same patients' unique identifiers were compared with the homeless service records, 18 of the 20 patients were identified as having experienced homelessness during the year. This might suggest that ED staff members' familiarity with certain frequent visitors may cause them to not document housing status or recognize the role that lack of housing may play in their medical conditions.

Homeless patients with complex medical issues face significant challenges in managing their health and securing consistent community-based care that helps stabilize their chronic and acute conditions and thus lessen the need for subsequent ED visits. Very-low-income individuals who are homeless tend to receive services at centers offering comprehensive care encompassing medical and behavioral health care as well as access to social services, but these services often fall short of providing stable housing.

\section{Limitations}

This study was limited to a small subset of total ED users. Care must be taken in generalizing to the larger group of frequent ED users and to frequent ED users in other areas. Data were not collected on admission rates or ED wait times, thereby limiting conclusions on ED and inpatient resource use among this population. Triage scores were used to suggest visit acuity but can only approximate actual acuity of illness. Triage methodology is different in each department; we attempted to ameliorate this by combining triage scores into broader categories. Coding may have been incomplete or inaccurate, limiting conclusions drawn about each patient's overall health. Care providers' familiarity with these individuals may have resulted in abbreviated documentation. Information on providers' awareness of these patients' use patterns and the impact of this awareness on clinical and resource decisions was not examined.

Our definition of homelessness was intentionally broad, including anyone who presented to one of two homeless service systems during the year of the study. It is not known if the two patients not identified as homeless simply did not use the mainstream homeless services that informed our study. Patients were not necessarily homeless at the time they presented to the ED.

Data from three EDs formed the basis of this study. These three departments lie within a few miles of each other, and they are not the only departments in the immediate area or the larger metropolitan region. Visits to other EDs would be unaccounted for in this study.

\section{Conclusion}

The frequent ED users identified in this study have significant medical problems. Their homelessness appears to be a unifying characteristic and may be a contributing factor to the frequency of their ED visits. Further evaluation of this patient group might demonstrate whether interventions such as intensive case management, access to housing programs that include health services, and targeted efforts to manage complex coexisting chronic diseases would improve the overall health of this population and decrease ED utilization.

\section{Conflict of Interests}

The authors have no commercial, financial, or other relationship related to the subject of this paper that would constitute or suggest a conflict of interest.

\section{Acknowledgments}

The paper was copyedited by Linda J. Kesselring, MS, ELS, the technical editor/writer in the Department of Emergency Medicine at the University of Maryland School of Medicine. The authors express special thanks to Arjun Chanmugam, MD, MBA, at Johns Hopkins Hospital, for expediting data acquisition at one study site and providing encouragement throughout this project.

\section{References}

[1] K. T. Xu, B. K. Nelson, and S. Berk, "The changing profile of patients who used emergency department services in the United States: 1996 to 2005," Annals of Emergency Medicine, vol. 54, no. 6, pp. 805-810, 2009.

[2] K. A. Hunt, E. J. Weber, J. A. Showstack, D. C. Colby, and M. L. Callaham, "Characteristics of frequent users of emergency departments," Annals of Emergency Medicine, vol. 48, no. 1, pp. $1-8,2006$.

[3] L. Moore, A. Deehan, P. Seed, and R. Jones, "Characteristics of frequent attenders in an emergency department: analysis of 1year attendance data," Emergency Medicine Journal, vol. 26, no. 4, pp. 263-267, 2009.

[4] K. K. Fuda and R. Immekus, "Frequent users of Massachusetts emergency departments: a statewide analysis," Annals of Emergency Medicine, vol. 48, no. 1, pp. 9-16, 2006.

[5] E. LaCalle and E. Rabin, "Frequent users of emergency departments: the myths, the data and the policy implications," Annals of Emergency Medicine, vol. 56, no. 1, pp. 42-48, 2010.

[6] Millions Still Face Homelessness in a Booming Economy, Urban Institute, Washington, DC, USA, 2000, http://www .urban.org/publications/900050.html. 
[7] G. Oates, A. Tadros, and S. M. Davis, "A comparison of national emergency department use by homeless versus non-Homeless people in the United States," Journal of Health Care for the Poor and Underserved, vol. 20, no. 3, pp. 840-845, 2009.

[8] R. E. Malone, "Whither the almshouse? Overutilization and the role of the emergency department," Journal of Health Politics, Policy and Law, vol. 23, no. 5, pp. 795-832, 1998.

[9] L. Solomon, C. Flynn, L. Eldred, E. Caldeira, M. P. Wasserman, and G. Benjamin, "Evaluation of a statewide non-name-based HIV surveillance system," Journal of Acquired Immune Deficiency Syndromes and Human Retrovirology, vol. 22, no. 3, pp. 272-279, 1999. 


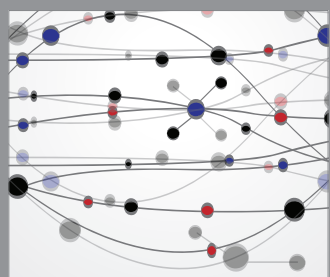

The Scientific World Journal
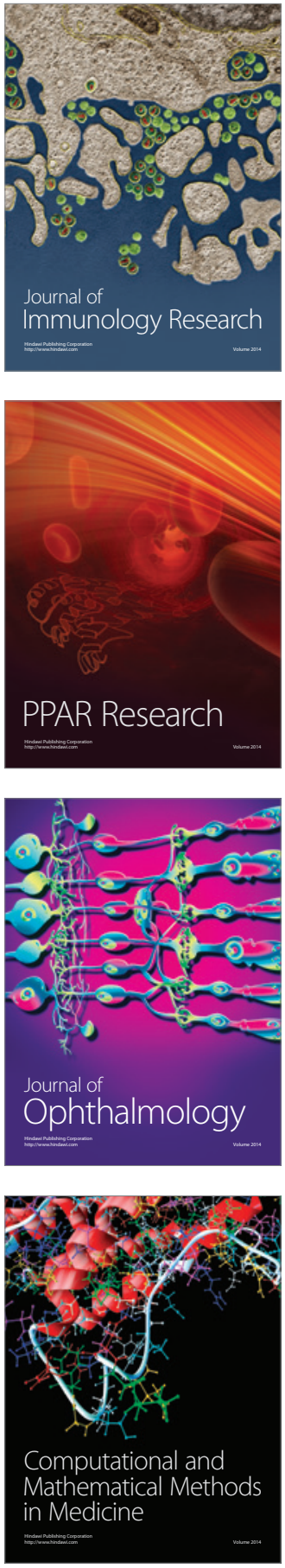

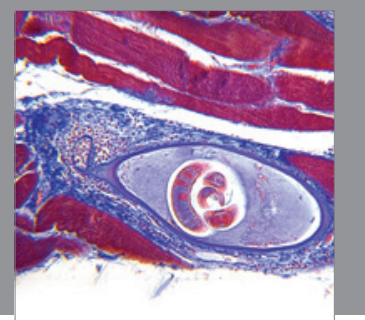

Gastroenterology

Research and Practice
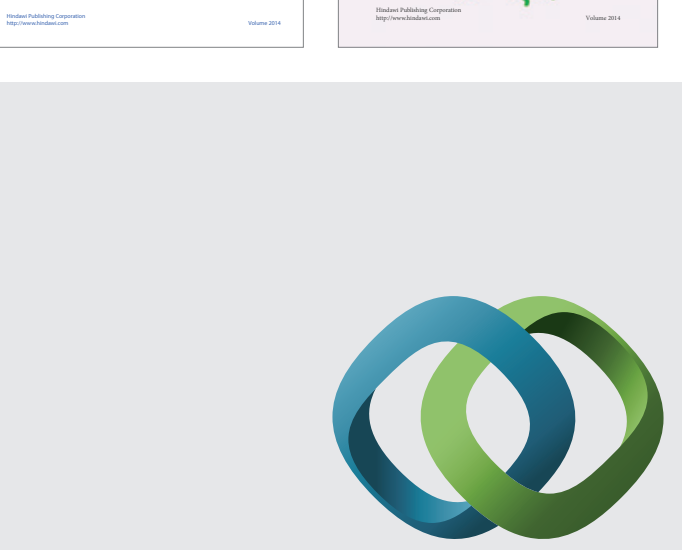

\section{Hindawi}

Submit your manuscripts at

http://www.hindawi.com
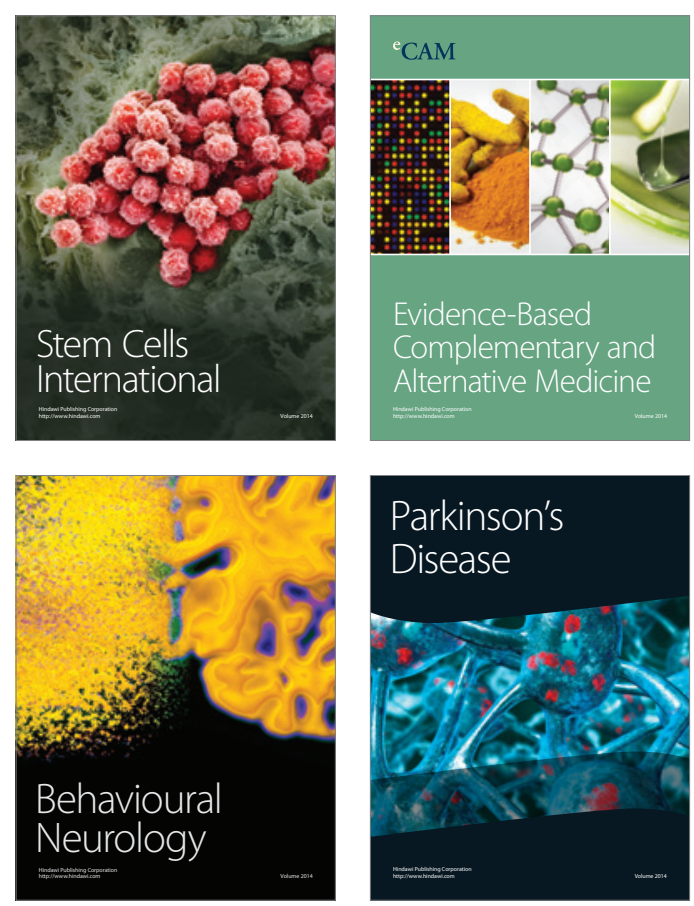

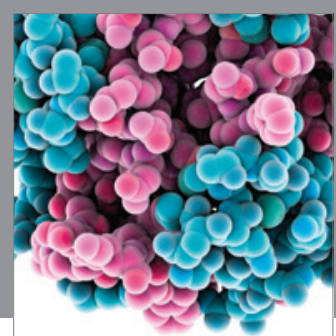

Journal of
Diabetes Research

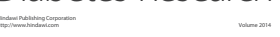

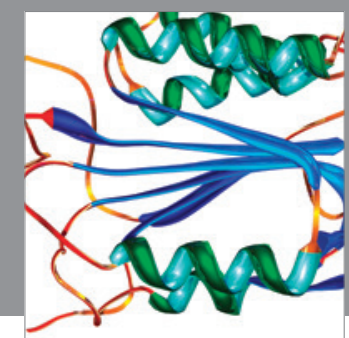

Disease Markers
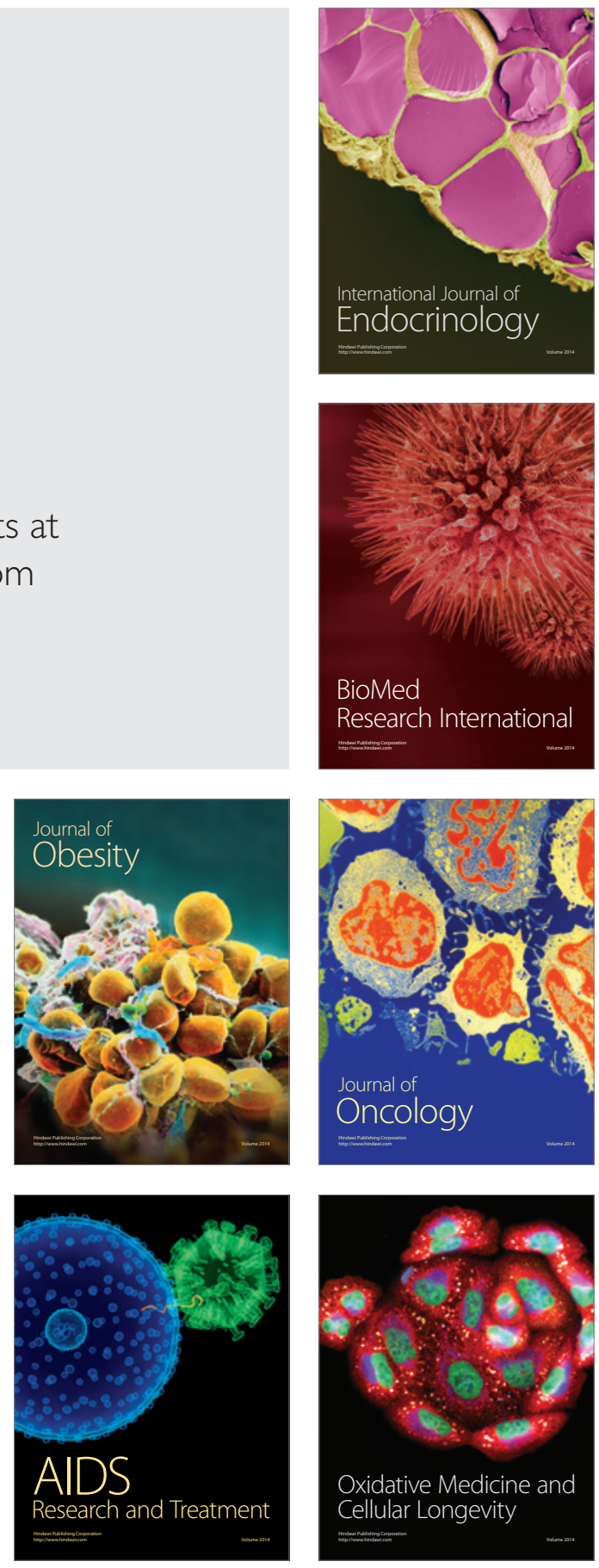